\title{
A!
}

This is an electronic reprint of the original article.

This reprint may differ from the original in pagination and typographic detail.

Shevchenko, Andriy; Kaivola, Matti; Javanainen, Juha

\section{Thermodynamics of a multicomponent atom sample in a tightly compressed atom trap}

Published in:

Physical Review A

DOI:

10.1103/PhysRevA.70.011403

Published: $28 / 07 / 2004$

Document Version

Publisher's PDF, also known as Version of record

Please cite the original version:

Shevchenko, A., Kaivola, M., \& Javanainen, J. (2004). Thermodynamics of a multicomponent atom sample in a tightly compressed atom trap. Physical Review A, 70(1), 1-4. [011403].

https://doi.org/10.1103/PhysRevA.70.011403

This material is protected by copyright and other intellectual property rights, and duplication or sale of all or part of any of the repository collections is not permitted, except that material may be duplicated by you for your research use or educational purposes in electronic or print form. You must obtain permission for any other use. Electronic or print copies may not be offered, whether for sale or otherwise to anyone who is not an authorised user. 


\title{
Thermodynamics of a multicomponent-atom sample in a tightly compressed atom trap
}

\author{
A. Shevchenko* and M. Kaivola \\ Department of Engineering Physics and Mathematics, Helsinki University of Technology, P. O. Box 2200, FIN-02015 HUT, Finland \\ J. Javanainen \\ Department of Physics, University of Connecticut, Storrs, Connecticut 06269-3046, USA \\ (Received 23 February 2004; revised manuscript received 30 April 2004; published 28 July 2004)
}

\begin{abstract}
We use classical statistical mechanics and thermodynamics to describe the response of a trapped multispecies atomic sample to a local deformation in the confining potential. An adiabatic deformation may not only increase the peak phase-space density, but also lower the temperature and spin-polarize the atoms.
\end{abstract}

DOI: $10.1103 /$ PhysRevA.70.011403

PACS number(s): 32.80.Pj, 05.70.Ce, 39.25.+k

The manipulation of atoms in microscopic potential wells is the technology presently regarded as the most likely foundation for practical applications of atom optics and BoseEinstein condensation (BEC) [1,2]. Well-localized magnetic [2-4] or electric [5,6] fields above the surface of a solid substrate have been successfully used to control the motion of microscopic atom samples [2,5] and to essentially simplify the creation of BEC $[2,7,8]$.

In regard to BEC, phase-space density is the key parameter. It cannot be increased by scaling the trapping potential $[9,10]$, but may be substantially adjusted by adiabatically deforming the trap [10-13]. This is also the case in evaporative cooling, where the trap is deformed in order to release atoms with a high kinetic energy. A similar increase of the phase space density is achieved by adding a tight potential to a wide trap. This method together with subsequent evaporation has been used to reach Bose-Einstein condensation of cesium [14] and, very recently, to create a two-dimensional BEC of cesium directly in a surface trap [15].

Phase space compression by a local change in the trapping potential has been studied theoretically for a single atomic species [11]. However, a given trap may carry more than one species, each of which may respond differently to the deformation. The present paper is based on our realization that allowing transitions between the Zeeman states of the atoms in conjunction with adjustments of the shape of an atom trap adds flexibility to the preparation of an atomic sample in a microtrap that could be part of an "atom chip" [1]. Such a chip could contain not only magnetic microtraps, but also static electric or all-optical traps. If, for instance, the chip substrate is made of material which is transparent to light, a gravito-optical surface trap [16-18] could serve as a container of cold atoms and as a trap to be modified locally.

In the present paper we use classical statistical mechanics and thermodynamics to investigate in general the properties of a multicomponent atom sample in a trap with locally deformed potential, allowing for both thermal and chemical equilibration. We demonstrate how an adiabatically driven deformation may decrease the temperature, increase the phase-space density of atoms in a particular magnetic state,

\footnotetext{
*Electronic address: andrej@focus.hut.fi
}

and spin-polarize atoms in an initially spin-independent atom trap.

Even though our method works for an arbitrary 3D atom trap, for illustration we consider a model for a surface trap so simple that the ensuing numerics can be handled with unsophisticated MATHEMATICA programs. Thus, the atoms are bound to a potential of the form $U(x)+U_{L}(y)+U_{h}(z)$, where $x, y$, and $z$ are Cartesian coordinates. The dependence on $z$ is assumed to be harmonic, $U_{h}(z)=m w_{0}^{2} z^{2} / 2$, with $m$ being the atomic mass and $w_{0}$ the trapping frequency in the direction perpendicular to the surface. In the $x$ and $y$ directions the atoms are confined to a slab of length $l$ and width $L$. We set $U_{L}(y) \equiv 0$, and $U(x)$ is a step function with the value $\Lambda$ added to a constant background in an interval of $x$ of length $\eta l$, with $\eta<1$. Overall, the potential inside the slab reads as $U_{t}(x, y, z)=m w_{0}^{2} z^{2} / 2+U(x)$. Figure 1 shows the trap boundaries and the potential $U_{t}(x, y, z)$ in a plane $(x, z)$ at a fixed value of $y$. Simplistic as the model is, we believe that it produces qualitatively correct results for the generic case when a potential is modified by an amount $\Lambda$ in a fraction $\eta$ of the trap volume.

We start by considering an ensemble of identical, but, in the sense of classical physics, distinguishable particles in the potential described above. As customary in kinetic theory for dilute gases, we ignore the effect of the interactions on the equilibrium. For $N$ particles the canonical partition function is

$$
Z_{N}=\frac{1}{N !}\left(\frac{1}{(2 \pi \hbar)^{3}} \int d^{3} r d^{3} p e^{-H / k_{B} T}\right)^{N},
$$

where $k_{B}$ is Boltzmann's constant, $T$ is the absolute temperature, and $H=\mathbf{p}^{2} / 2 m+U_{t}$ is the one-particle Hamiltonian.

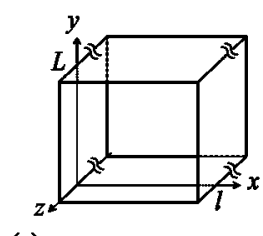

(a)

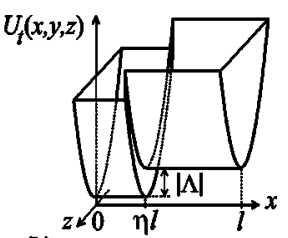

(b)
FIG. 1. (a) The trap boundaries in the $x$ and $y$ directions. (b) The trapping potential, $U_{t}(x, y, z)$, in a plane $(x, z)$ at a fixed $y$ from the interval $0<y<L$. In the sketch, $\Lambda$ is negative. 
Helmholtz free energy $F=-k_{B} T \ln Z_{N}$ is found to be

$$
F=N k_{B} T\left(\ln \left[\frac{\rho(T)}{\int_{0}^{l}(d x / l) e^{-U(x) / k_{B} T}}\right]-1\right),
$$

where

$$
\rho(T)=\frac{2 \pi \hbar^{3} w_{0} N}{k_{B}^{2} T^{2} m l L}
$$

is the usual phase space density at the bottom of the confining potential in the absence of the deformation, and

$$
n(T)=\sqrt{\frac{m}{2 \pi k_{B} T}} \frac{w_{0} N}{l L}
$$

is the corresponding density. The final -1 in Eq. (2) originates from the Stirling expansion as in $\ln N !=N \ln N-N$. The entropy $S=-\partial F / \partial T$ is

$$
S=N k_{B}\left(3-\ln \left[\frac{\rho(T)}{\int_{0}^{l}(d x / l) e^{-U(x) / k_{B} T}}\right]+\frac{\left\langle U_{x}\right\rangle}{k_{B} T}\right),
$$

where $\left\langle U_{x}\right\rangle$ is the thermal expectation value of the potential $U(x)$. The chemical potential is found as $\mu=\partial F / \partial N$. For the stepwise shape of $U(x)$, we derive the following analytical expressions for one-particle dimensionless entropy $s$ $=S / N k_{B}$, mean energy per particle expressed in units of temperature $\epsilon=(F+T S) / N k_{B}$, and chemical potential $\xi=\mu / k_{B}$ similarly in units of temperature,

$$
\begin{gathered}
s=3-\ln \frac{\rho(T)}{1-\eta+\eta e^{-\Delta / T}}+\frac{\eta e^{-\Delta / T} \Delta}{T\left(1-\eta+\eta e^{-\Delta / T}\right)}, \\
\epsilon=\epsilon_{0}+2 T+\frac{\eta e^{-\Delta / T} \Delta}{1-\eta+\eta e^{-\Delta / T}}, \\
\xi=\epsilon_{0}+T\left(\ln \frac{\rho(T)}{1-\eta+\eta e^{-\Delta / T}}-1\right) .
\end{gathered}
$$

Here $\Delta=\Lambda / k_{B}$ is the local change of the potential and $\epsilon_{0}$ the potential at the bottom of the nondeformed trap, both also in units of temperature. The phase space density at the potential minimum within the deformation and the corresponding density are obtained from

$$
\begin{aligned}
& \rho(T, \Delta, \eta)=\frac{\rho(T)}{\eta+(1-\eta) e^{\Delta / T}}, \\
& n(T, \Delta, \eta)=\frac{n(T)}{\eta+(1-\eta) e^{\Delta / T}},
\end{aligned}
$$

with $\rho(T)$ and $n(T)$ defined in Eqs. (3) and (4).

In the case of a single atomic species in the trap, an adiabatic change of the potential is described by

$$
s\left(T_{i}, \Delta_{i}, \eta_{i}\right)=s\left(T_{f}, \Delta_{f}, \eta_{f}\right),
$$

which is the requirement for conservation of entropy. The indices $i$ and $f$ denote the initial and final values of the vari- ables, respectively. By solving Eq. (11), one can find $T_{f}$, and subsequently, all other thermodynamic parameters of the system.

We proceed to the case in which two or more atomic species $k$, each in thermal equilibrium in its own locally deformed potential well, are allowed to exchange energy but not particles. The final equilibrium temperature $T_{f}$ of the whole system is then calculated by equating the initial and final total energies of the system. On the other hand, if the deformations are varied adiabatically starting from a common temperature, the final temperature is found by equating the entropy of the whole system before and after the transformation,

$$
\sum_{k} s_{k}\left(T_{i}, \Delta_{k i}, \eta_{k i}\right) N_{k}=\sum_{k} s_{k}\left(T_{f}, \Delta_{k f}, \eta_{k f}\right) N_{k} .
$$

Next we think of atoms in a particular quantum state as a species that can exchange particles with the other species. Zeeman states in an angular-momentum degenerate energy level serve as an example. Equilibration between the species can be controlled with rf driving fields, or with laser driven Raman transitions. Inasmuch as the rotating wave approximation of quantum optics is applicable, the detunings set the relative energies of the states for the purposes of thermodynamics.

Again we start by considering the case when each species is initially in its own equilibrium at a temperature $T_{k i}$, whereafter the exchanges of energy and particles equilibrate the whole system. Since the system as a whole is closed, the total energy is conserved. We therefore write

$$
\sum_{k} \epsilon_{k}\left(T_{k i}, \Delta_{k}, \eta_{k}\right) N_{k i}=\sum_{k} \epsilon_{k}\left(T_{f}, \Delta_{k}, \eta_{k}\right) N_{k f} .
$$

The final state will contain species with equal chemical potentials. We have

$$
\begin{gathered}
\xi_{k}\left(T_{f}, \Delta_{k}, \eta_{k}\right)=\xi_{k^{\prime}}\left(T_{f}, \Delta_{k^{\prime}}, \eta_{k^{\prime}}\right), \\
\sum_{k} N_{k f}=N,
\end{gathered}
$$

where $k$ and $k^{\prime}$ denote different species, and $N$ is the conserved total number of atoms. The final temperature $T_{f}$ and the atom numbers for each species $N_{k f}$ are found by solving the coupled equations (13)-(15).

Yet another situation occurs if the local deformations of the states evolve adiabatically in an already equilibrated system. Then the chemical potentials of the subensembles will be equal to each other at each moment of the transformation and the entropy of the whole system will be conserved. Hence, the equations for calculation of $T_{f}$ and $N_{k f}$ are

$$
\xi_{k}\left(T_{f}, \Delta_{k f}, \eta_{k f}\right)=\xi_{k^{\prime}}\left(T_{f}, \Delta_{k^{\prime} f}, \eta_{k^{\prime} f}\right),
$$

$$
\sum_{k} N_{k f}=N_{t}
$$




$$
\sum_{k} s_{k}\left(T_{i}, \Delta_{k i}, \eta_{k i}, N_{k i}\right) N_{k i}=\sum_{k} s_{k}\left(T_{f}, \Delta_{k f}, \eta_{k f} N_{k f}\right) N_{k f}
$$

Now that the procedure for solving $T_{f}$ and $N_{k f}$ in different thermodynamic processes has been laid down, we proceed to applications. We first consider the possibility to reach a lower temperature in laser cooling by introducing a small potential well in the trap during the cooling stage and adiabatically removing the well after the cooling is complete. This method can be applied if the cooling efficiency does not change in the presence of the subtrap and if the cooled sample can reach thermal equilibrium before the cooling mechanisms are switched off. These conditions can be satisfied, e.g., in evanescent-wave cooling of atoms in a GOST [12,15-18]. In fact, a "dimple" subtrap created in a GOST with an infrared focused laser beam is frequently employed $[12,15]$. Figure 2(a) shows the final temperature, $T_{f}\left(\Delta_{i}, \eta\right)$, that can be achieved by using a subtrap of initial depth $\left|\Delta_{i}\right| \quad\left(\Delta_{i}<0\right)$ and effective volume characterized by $\eta$. The calculations are done with the aid of Eq. (11), where $\Delta_{f}$ is set to 0 and both $\eta_{i}$ and $\eta_{f}$ are replaced with the same parameter $\eta$. For generality, we use the dimensionless variables $\eta$ and $\left|\Delta_{i}\right| / T_{0}$, where $T_{0}$ is the initial temperature $\left[T_{0} \equiv T_{i} \equiv T_{f}\left(\Delta_{i}\right.\right.$ $=0)$ ]. The calculations show that after cooling of the atoms in the presence of a small subtrap, the temperature may be decreased by an additional order of magnitude by slowly switching the subtrap off. The method allows reduction of the temperature below the recoil limit, a problematic task for laser cooling alone. We note that in general the use of a smaller but deeper subtrap will allow the temperature to decrease to a lower level. However, depending on the total number of atoms, this can lead to an inappropriately high loss rate of the trapped atoms due to inelastic two- and threebody collisions. The calculated curves for $T_{f} / T_{0}$ help to find optimum values for the parameters $\eta$ and $\Delta_{i}$.

In the next example we calculate the peak phase space density of atoms in a trap with an adiabatically inserted subtrap [11]. The peak phase space density $\rho\left(\Delta_{f}, \eta\right)$ related to its original value $\rho_{0}=\rho\left(\Delta_{f}=0\right)$ is shown in Fig. 2(b) as a function of $\eta$, volume ratio, and $\left|\Delta_{f}\right| / T_{0}$, the final depth of the subtrap over the initial temperature. The calculations are carried out using Eq. (11) with $\Delta_{i}=0$ and $\eta_{i}=\eta_{f} \equiv \eta$ to find $T_{f}$, and Eq. (9) to find $\rho\left(T_{f}, \Delta_{f}, \eta\right) \equiv \rho\left(\Delta_{f}, \eta\right)$. The results agree well with the experiments of Ref. [12], where the phase space density was locally increased by two orders of magnitude using a "dimple" trap within a GOST. It can be seen that at each fixed value of $\eta$, there is an optimum value of $\left|\Delta_{f}\right| / T_{0}$, at which the phase-space density reaches its maximum. Above this value, the number of atoms transferred into the subtrap becomes too large, so that the rest of the atoms in the trap can no longer keep the temperature at a low enough level.

We proceed to the case when a spin-independent atom trap is locally deformed with a magnetic field. In a dipole trap with, e.g., spin-unpolarized ${ }^{87} \mathrm{Rb}$ in the lower hyperfine ground state, the magnetic field splits up the sample into three species characterized by the magnetic quantum num-
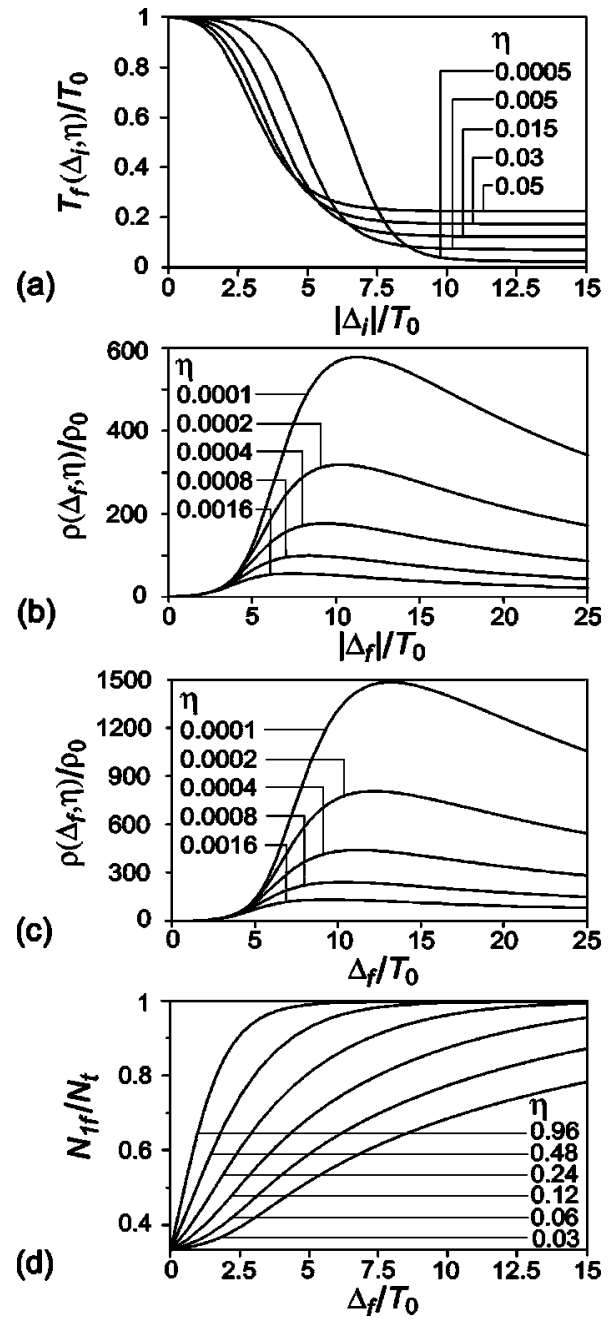

FIG. 2. (a) A decrease of temperature in an atom trap caused by the adiabatic removal of a subtrap. (b) An increase of the peak phase-space density of trapped atoms caused by adiabatic insertion of a subtrap. (c) An increase of the peak phase-space density of atoms in a particular magnetic state due to adiabatic deformation of the trap with a static magnetic field. (d) The spin polarization of atoms in a dipole trap due to adiabatic deformation of the trapping potential with a static magnetic field.

bers $m_{F}=\{-1,0,+1\}$. When the trap is deformed with a locally high magnetic field, the atoms with $m_{F}=+1$ are attracted to the deformation, while atoms with $m_{F}=-1$ are repelled from it. The deformation can also be created by using a magnetic-field minimum which will attract $m_{F}=-1$ atoms. Independently of the deformation type, there will be three species $(k=1,2,3)$ with $\Delta_{k i}=0$ and $\Delta_{k f}=\left\{-\Delta_{f}, 0,+\Delta_{f}\right\}$, where now $\Delta_{f}$ is chosen to be positive. All the parameters $\eta_{k i}$ and $\eta_{k f}$ are set to the same value $\eta$.

Let us denote the initial temperature by $T_{0}$, and the initial phase-space density, equal for all species, by $\rho_{0}$. We calculate the enhancement of the phase-space density, $\rho\left(T_{f}\right.$, $\left.-\Delta_{f}, \eta\right) / \rho_{0}$, for the species with $\Delta_{k f}=-\Delta_{f}$, considering three different cases. In the first case we assume that the exchange of energy between the species occurs much faster than particle exchange, and ignore the latter altogether. We simply apply the conservation of entropy in Eq. (12). In the second 
case, we allow the equilibration of atom numbers to follow the equilibration of temperature. We solve Eq. (12), and then replace all $T_{k i}$ in Eqs. (13) and (14) with the obtained $T_{f}$. Then, solving Eqs. (13)-(15), we find a new $T_{f}$ and new atom numbers $N_{k f}$. In the third case we allow the system to be in total equilibrium (thermal and chemical) at each moment of the transformation, and use Eqs. (16)-(18) directly. When the phase space density increases substantially for the species attracted toward the deformation, $\rho\left(T_{f},-\Delta_{f}, \eta\right) \gg \rho_{0}$, the results for the three scenarios turn out to be almost indistinguishable. In Fig. 2(c) we show a plot corresponding to the first case. The dependence of $\rho\left(T_{f},-\Delta_{f}, \eta\right) / \rho_{0}$ on the parameters $\eta$ and $\Delta_{f} / T_{0}$ is similar to the dependence illustrated in Fig. 2(b) for a single species. However, here the factor by which the phase space density increases is about three times larger, as if the sample was spin-polarized at the temperature $T_{0}$ prior to applying the magnetic field.

Our final example may be of interest to those who are searching for ways to spin-polarize atoms without increasing the temperature. Let atoms in a ground state with three magnetic substates be stored in a dipole trap in the presence of an external magnetic field. The constant magnetic field splits the initial degeneracy of the species. However, if a rf field in resonance with the splitting is used to accelerate the equilibration between the species, the degeneracy is restored as far as it comes to thermodynamics. Next, a local magnetic-field deformation is inserted adiabatically. To calculate the final atom numbers $N_{k f}$ of the three magnetic states, we solve Eqs. (16)-(18) with $\eta_{k i}=\eta_{k f} \equiv \eta, \Delta_{k i}=0$, and $\Delta_{k f}=\left\{-\Delta_{f}, 0,+\Delta_{f}\right\}$. The dependence of the normalized population $N_{1 f} / N_{t}(k=1$ corresponds to the state deformed by $-\Delta_{f}$ ) on $\eta$ and $\Delta_{f} / T_{0}$ is plotted in Fig. 2(d). The result shows that the use of a deformation with a large relative volume, $\eta$, and/or a large relative strength, $\Delta_{f} / T_{0}$, can provide nearly complete spin polarization of the atoms. By switching off the rf field and slowly removing the deformation, one can then return the trap to its initial shape and the sample to its initial temperature. We note that decoupling of the magnetic states by switching off the rf field leads to an essential change of the system, because the rest of the transformation proceeds without equilibration of the magnetic-state populations. The disappearance of the rf field does not affect the spatial profiles of the magnetic energy levels and, therefore, it does not change the temperature.

In conclusion, we have proposed a simple theoretical model based on classical statistical mechanics to describe a trapped sample of atoms in the presence of an adiabatically varying local deformation of the confining potential. Such a deformation can substantially increase the peak phase space density $[10,11]$. In regard to applying the model to integrated atom chips [1] we have noted here that a rich variety of new ways to manipulate the sample opens up by making use of the magnetic substates of an atom. Examples are presented on increasing the phase phase density, on atom cooling, and on polarizing the atomic spins.

We acknowledge financial support from the Academy of Finland, NSF, and NASA. One of us (J.J.) is particularly grateful to the Helsinki University of Technology for the hospitality extended to him during the initial phases of this work.
[1] R. Folman, P. Krüger, J. Schmiedmayer, J. Denschlag, and C. Henkel, Adv. At., Mol., Opt. Phys. 48, 263 (2002).

[2] J. Reichel, Appl. Phys. B: Lasers Opt. 75, 469 (2002).

[3] D. Cassettari et al., Appl. Phys. B: Lasers Opt. 70, 721 (2000).

[4] M. Vengalattore, W. Rooijakkers, and M. Prentiss, Phys. Rev. A 66, 053403 (2002).

[5] P. Krüger et al., Phys. Rev. Lett. 91, 233201 (2003).

[6] S. K. Sekatskii, B. Riedo, and G. Dietler, Opt. Commun. 195, 197 (2001).

[7] W. Hänsel, P. Hommelhoff, T. W. Hänsch, and J. Reichel, Nature (London) 413, 498 (2001).

[8] S. Schneider et al., Phys. Rev. A 67, 023612 (2003).

[9] W. Ketterle and D. E. Pritchard, Phys. Rev. A 46, 4051 (1992).
[10] P. W. H. Pinkse et al., Phys. Rev. Lett. 78, 990 (1997).

[11] D. M. Stamper-Kurn et al., Phys. Rev. Lett. 81, 2194 (1998).

[12] M. Hammes, D. Rychtarik, H.-C. Nägerl, and R. Grimm, Phys. Rev. A 66, 051401 (2002).

[13] L. Viverit, S. Giorgini, L. P. Pitaevskii, and S. Stringari, Phys. Rev. A 63, 033603 (2001).

[14] T. Weber et al., Science 299, 232 (2003).

[15] D. Rychtarik, B. Engeser, H.-C. Nägel, and R. Grimm, e-print cond-mat/0309536.

[16] M. Hammes et al. J. Mod. Opt. 47, 2755 (2000).

[17] Yu. B. Ovchinnikov, I. Manek, and R. Grimm, Phys. Rev. Lett. 79, 2225 (1997).

[18] J. Söding, R. Grimm, and Yu. B. Ovchinnikov, Opt. Commun. 119, 652 (1995). 